\title{
Téoros
}

Revue de recherche en tourisme

\section{Hôtellerie et développement régional}

Réflexions autour de paradoxes

\section{Normand Cazelais}

Volume 23, numéro 3, automne 2004

Regards sur l'hôtellerie : un secteur en mutation

URI : https://id.erudit.org/iderudit/1071227ar

DOI : https://doi.org/10.7202/1071227ar

Aller au sommaire du numéro

Éditeur(s)

Université du Québec à Montréal

ISSN

0712-8657 (imprimé)

1923-2705 (numérique)

Découvrir la revue

Citer cet article

Cazelais, N. (2004). Hôtellerie et développement régional : réflexions autour de paradoxes. Téoros, 23(3), 17-21. https://doi.org/10.7202/1071227ar d'utilisation que vous pouvez consulter en ligne.

https://apropos.erudit.org/fr/usagers/politique-dutilisation/ 


\section{Hôtellerie et développement régional Réflexions autour de paradoxes}

\section{Normand Cazelais}

Conrad Nicholson Hilton, fondateur de la chaîne hôtelière de luxe à son nom qui compte aujourd'hui plus de 500 établissements dans le monde, avait coutume de répéter que les trois principaux facteurs de succès dans ce domaine sont le site, le site et encore le site. Au-delà de son humour, cette opinion vaut d'être retenue. Le lien entre l'hôtellerie et la géographie est de grande importance. Le rôle que joue la première dans le développement régional est par contre assez délicat à circonscrire même si, en beaucoup de régions, la tentation est forte de se tourner vers le tourisme et, par voie de conséquence, vers l'hôtellerie pour requinquer une économie en difficulté. Sur cet aspect, toutefois, les études tant de l'OCDE que de l'OMT ou de la Banque mondiale ne manquent pas pour démontrer que la création d'emplois y est plus rapide et moins onéreuse que dans d'autres secteurs d'activités, pour illustrer également qu'il est inquiétant de voir une économie se construire sur le tourisme en raison de sa fragilité structurelle.

Textes et études abondent pour traiter de la place que tient l'hôtellerie dans le tourisme et l'économie, entre autres pour analyser les métiers et les moyens à mettre en œuvre afin d'assurer la meilleure formation possible du personnel, pour évaluer la performance des établissements ou pour traiter de la distribution, du marketing, de la gestion, de la fiscalité, des relations de travail et de l'écologie. Les écrits sont par contre fort discrets sur le lien entre hôtellerie et développement régional, surtout dans sa dimension spatiale. Trois textes, puisés à des univers fort dif-

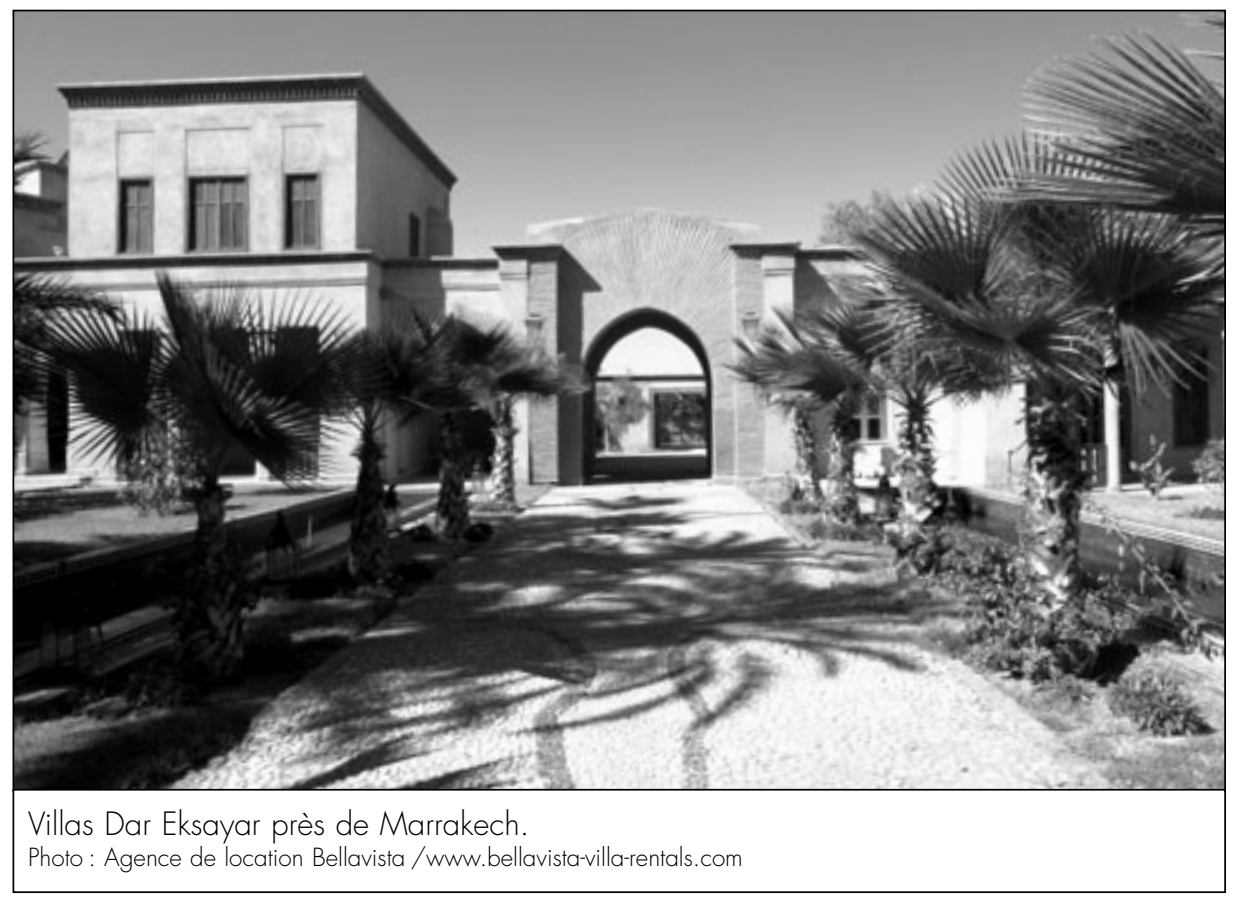

férents mais portant chacun sur le rôle économique du tourisme, peuvent illustrer ce propos (Moreau, 2003 ; WTTO, 1993 ; Paci, 1993). Pourtant, l'hôtellerie génère des retombées directes et indirectes qui peuvent contribuer pour beaucoup - non sans paradoxes toutefois - au dynamisme économique des régions, à leur organisation et à l'aménagement de leurs espaces respectifs. C'est ce que nous aborderons dans les lignes qui suivent.

\section{Le rôle stratégique de l'hôtellerie}

L'investissement dans le secteur hôtelier pour en augmenter la capacité constitue la plupart du temps l'aune de mesure de l'effort consenti dans un territoire national ou régional pour développer le tourisme.
Voyons un exemple contemporain, celui du Maroc. Depuis le 10 janvier 2001, date du «discours royal» de Marrakech, ainsi appelé car il fut prononcé par le roi Mohammed VI, autorité suprême du pays. Ce pays d'Afrique du Nord baigné par les eaux de l'Atlantique et de la Méditerranée a fait du tourisme une «priorité nationale». A alors été signé un «contrat-programme » entre l'État, le secteur privé et les régions - un « accord historique »-dont l'objectif est d'attirer 10 millions de touristes étrangers en 2010 , volume qui est présentement de 4,4 millions par an.

La stratégie pour atteindre ce résultat repose sur un stock hôtelier à diversifier et dont il faudra faire passer la capacité de 99000 à 230000 lits, pour ensuite porter le nombre de nuitées de 10 à 50 millions et les recettes 


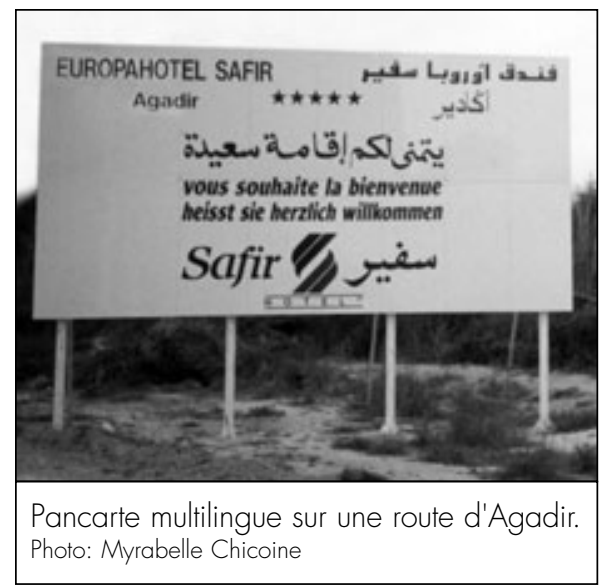

de 24 milliards de dirhams (environ 3 milliards de dollars) à 80 milliards de dirhams (un peu plus de 11 milliards de dollars). Au cœur de cette action figure le plan Azur, véritable programme national de développement régional, qui propose la création de six stations balnéaires d'envergure.

Épine dorsale du programme 2010, le plan accorde un poids tout particulier au tourisme balnéaire par l'aménagement d'une station sur la Méditerranée (Saïdia) et de cinq autres sur l'Atlantique (Taghazout, Mogador, Mazagan, Lixus et Plage Blanche). L'intention avouée est de miser, par de substantiels investissements, sur un «balnéaire intelligent, écologique, de convivialité », en « rupture » avec celui qui a reposé jusqu'ici sur le sable et le soleil. Aux yeux des autorités, cet « effort» sur le littoral devrait profiter à l'arrière-pays tout en appuyant le fort attrait touristique qu'exercent Marrakech, Fès et Méknès, les villes impériales de l'intérieur, depuis longtemps inscrites sur les itinéraires des voyageurs.

Déjà à Agadir, dont la baie s'inscrit dans le très sélect club des trente plus belles baies du monde, des effets sont «tangibles » sur le réseau d'eau potable et d'assainissement. Articulé autour de l'hôtellerie, le tourisme doit agir, dans l'esprit des planificateurs et des dirigeants marocains, comme levier sur l'essor de la région en soutenant le réaménagement du port de pêche et de la casbah, l'apparition de nouveaux festivals et la revalorisation des campagnes environnantes.

\section{Emplacement et fonctions}

Comme son nom l'indique, le développement régional ajoute une connotation géographique au concept de développement économique :

Le concept de région est [...] maintenant au centre de toute discussion sur l'aménagement du territoire. [...] Il s'agit là d'un mouvement général, qui depuis 1945 joint dans certains cas à des facteurs historiques, a enfanté ou ressuscité les länder allemands, les régions autonomes italiennes, les circonscriptions régionales françaises, les régions britanniques de planification, etc. (Monod et de Castelbajac, 1971 : 40).

Une région est «une unité territoriale de localisation, de dimensions et de formes diverses; [elle] fait référence à une partie d'un ensemble plus vaste qui s'impose à l'observation comme la combinaison de multiples facteurs » (Encyclopédie Bordas, 1994 : 4280). La région est elle-même composée d'unités spatiales plus petites qui, assemblées et interreliées, lui donnent son identité. Puisqu'ils occupent chacun un lieu particulier, c'est à cette dernière échelle - qui inclut celle des sites - que se relient les établissements hôteliers. Notons qu'un hôtel était, à l'origine, la résidence d'un grand personnage, un roi le plus souvent. La montée de la bourgeoisie a entraîné l'apparition de résidences cossues, rivalisant d'aisance, qui ont été édifiées dans les villes : avec l'apparition des premiers hôtels dûment destinés aux voyageurs, on les a appelés hôtels particuliers. Quant aux hôtels de ville, ils constituent le siège physique de l'autorité municipale; en Europe, notamment, ce sont de véritables monuments architecturaux, hauts lieux de l'espace de leur localité respective. En certains endroits, comme à Eschalens en Suisse, l'hôtel de ville fait aussi fonction d'hôtel pour voyageurs.

Par définition, un voyageur est une personne qui se déplace entre deux points géographiques. En chemin, il a besoin d'hébergement, service qui doit, par conséquent, se trouver sur son trajet. C'est pourquoi les hôtels, du moins dans leur fonction originelle de relais, sont au voisinage des ports, des gares, des routes, des autoroutes et des aéroports, à l'entrée des agglomérations ou en leur centre. Par la suite, suivant le développement des activités de loisir, plusieurs s'implantent en bord de rivières et de lacs, en montagne au pied de centres de ski, près de terrains de golf, de plages, de parcs, etc.

La localisation des établissements hôteliers évolue donc, tout comme leur contribution au développement régional. Petit à petit, la fonction originelle de support de l'hôtellerie s'est doublée d'une fonction d'appel, comme en attestent les hôtels de villégiature et les autres resorts qui intègrent de multiples activités sportives ou culturelles et deviennent, par le fait même, de véritables stations touristiques, parfois nettement coupées du support physique et socioculturel environnant. À l'exemple du Club Med, les hôtels « tout compris », connus sous l'appellation de Super Clubs, qui ont d'abord fait florilège dans les Antilles, constituent des destinations dans la destination : ainsi, les vacanciers ne vont pas tant en Jamaïque mais, en particulier, à Sandals, à Couples et à d'autres Hedonism.

Pour connaître popularité et prospérité, les hôtels de destination et, à plus forte raison, les resorts, ont besoin, outre une main-d'œuvre suffisante et qualifiée, d'infrastructures de services adéquates: eau potable en qualité et en quantité, traitement efficient des eaux usées, alimentation constante en énergie, etc. $\mathrm{Ce}$ besoin est particulièrement affirmé en matière de transport; il exige une desserte efficace par la route, le rail, les voies fluviales, maritimes ou aériennes ou, mieux encore, par une combinaison de ces différents moyens, autant d'éléments qui contribuent activement à structurer le développement régional. À leur tour, ces établissements vont provoquer l'apparition d'une gamme plus ou moins vaste et complexe d'équipements connexes tels que réseaux voyers, centres commerciaux, aires résidentielles et pôles de services. 


\section{Le cas de Charlevoix'}

À la rencontre spectaculaire d'un fleuve qui est déjà une mer et de montagnes qui s'y jettent abruptement, Charlevoix a longtemps vécu de cabotage, puis d'agriculture et d'exploitation forestière. Au dix-huitième siècle est apparue une villégiature estivale, structurée autour de villas cossues qui ont permis l'implantation d'un landmark de l'hôtellerie au Québec, à savoir le Manoir Richelieu. Ce premier tourisme fut soutenu par la présence du chemin de fer et surtout par la prospérité des navires de croisière - pensons, entre autres, aux «bateaux blancs » de la Canada Steamship Line - qui ont sillonné le Saint-Laurent et le Saguenay jusqu'aux années soixante.

Avec l'expansion de l'automobile et du réseau routier, rails et croisières ont perdu leur popularité et cette forme de tourisme a connu le déclin. Plusieurs villas ont été délaissées et le manoir Richelieu lui-même a dû fermer ses portes. Pendant presque deux décennies, le tourisme dans Charlevoix a connu un état de latence, sinon de crise.
Un net regain s'est fait sentir à la fin des années soixante-dix avec l'émergence d'un produit nouveau : les auberges.

Remarquable par sa diversité, son nombre et son rayonnement, le réseau des auberges a changé la donne en contribuant à refaire de Charlevoix une destination [...] Le renouveau et l'éclosion des auberges ont permis d'y allonger les séjours. Et de constater qu'au-delà des beaux paysages il y avait une vie, une culture, un patrimoine, des gens. Pas seulement en été mais en toutes saisons (Cazelais, 1998: 51-52).

Au sein d'une économie qui s'essoufflait en raison du peu de vitalité de l'agriculture, de la mise au rencart des goélettes et d'une stagnation de la foresterie, les auberges ont donné le coup de pouce nécessaire au tourisme pour qu'il devienne tout au cours de l'année un actif important, voire essentiel, du développement de la région de Charlevoix : à compter du tournant des années quatre-vingt, Charlevoix a affiché

\section{Le cas du Club Med}

La localisation et la distribution des Clubs Méditerranée sont intimement liées aux qualités intrinsèques des sites d'accueil. Depuis ses origines, l'entreprise sélectionne avec grand soin les lieux où s'établissent ses "villages », préférablement dans des parties du monde recherchées par les vacanciers ou porteuses d'un fort exotisme (Antilles, Tahiti, Seychelles, etc.). L'image de beauté est le premier critère, puisque le Club vend du rêve. Pour les destinations soleil, il faut une mer splendide, une plage d'une exceptionnelle qualité, un décor de carte postale, une superficie assurant tranquillité et réclusion. L'équivalent est également recherché pour la neige et la montagne.

D'ailleurs, le terme "village» traduit bien l'intention initiale: il s'agit d'un espace convivial, «tribal » en quelque sorte, qui reprend et améliore, à l'échelle du microcosme, ce qui existe dans la société et dans la nature. Pour l'essentiel, c'est un espace prédéfini en fonction de la clientèle - les "gentils membres » ou GM - en vacances : il y fait bon vivre, les contraintes de la vie quotidienne y sont reléguées aux oubliettes, grâce au cadre préalablement qualifié d'enchanteur et aux bons soins des "gentils organisateurs » ou GO. L'accent est - ou était ? - mis sur l'appartenance, le temps d'une période d'évasion, à un espace plus ou moins clos et à une collectivité dont les membres ne se connaissent pas, mais qui en acceptent les règles fondées sur le jeu, la détente, l'absence de tracas et de responsabilités.

Les sites seront aménagés en conséquence : clôtures ou no man's land entourant la propriété pour affirmer la distinction avec l'espace environnant et le tissu social ambiant; aires d'hébergement (souvent des " huttes » ou des villas) nettement délimitées, séparées des zones vouées au farniente, à la pratique des sports, à la danse, etc. par de vastes espaces de rencontre où buffets et tables grand format tiennent un rôle privilégié. Qui plus est, tout est compris dans le prix du forfait : le GM appartient alors à un autre monde, à un espace autosuffisant, assimilé à une forte plus-value.

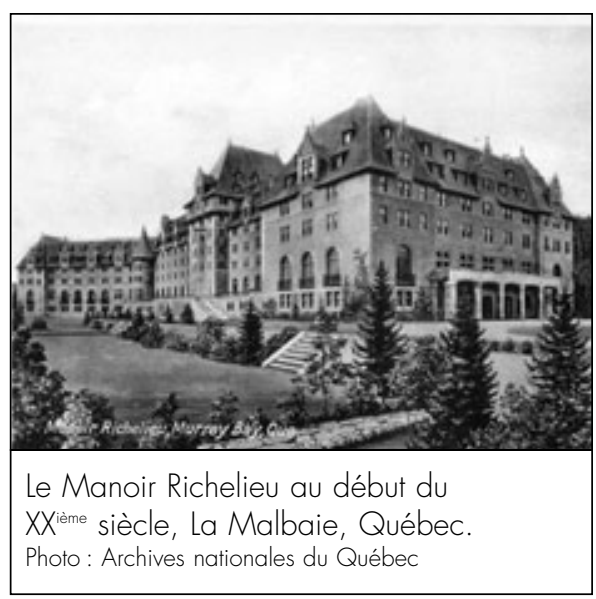

une nouvelle prospérité et les auberges y ont été pour beaucoup. En recyclant des villas huppées qui avaient connu de meilleurs jours, elles ont imprimé une image de marque à la région, une image haut de gamme liée à un indéniable patrimoine architectural et culturel.

Le plein potentiel touristique de Charlevoix pouvait enfin s'exprimer. Le Manoir Richelieu a été ranimé de ses cendres au point de faire aujourd'hui partie de la prestigieuse famille des hôtels Fairmont et d'accueillir un casino d'envergure internationale, le train passagers est revenu, Baie-Saint-Paul a exhibé ses trésors, la villégiature s'est transformée et s'est déployée, le corpus architectural a retrouvé son lustre, l'agriculture s'est largement redéfinie dans le sillon des produits du terroir. Le produit touristique lui-même s'est diversifié avec l'éclosion d'une vaste gamme d'activités et d'équipements axés sur le paysage bien sûr, mais également sur le ski nordique et alpin, sur l'histoire, la peinture, la musique et les arts en général, sur le plein air et la gastronomie. En attestent la Route des saveurs, les divers circuits des peintres, des artisans, des plus beaux paysages et panoramas, des patrimoines religieux, maritime et culturel ou les autres activités telles que l'International de l'art vocal de Charlevoix, le Festival international du domaine Forget ou le Symposium international d'art contemporain de Baie-Saint-Paul.

Ainsi, s'est concrétisée dans Charlevoix la relation entre le développement régional et l'affirmation originale d'un segment de l'hôtellerie. 


\section{Le cas des Laurentides de Montréal}

Dans les Laurentides de Montréal $^{2}$, la venue du chemin de fer du Canadian Pacific Railway puis de la route 11 , aujourd'hui la route 117, dans le sillon de la vallée de la rivière du Nord a entraîné, en certains points de chute dotés d'attraits liés à des plans d'eau ou à des aires skiables, l'émergence d'un chapelet d'hôtels renommés dont la clientèle se recrutait pour une large part à l'extérieur même du Québec: ce furent, par exemple, le Tremblant Lodge et le Gray Rocks à Mont-Tremblant, le Chantecler à Sainte-Adèle, l'Alpine Inn à Sainte-Marguerite Station, La Sapinière à Val-David. L'implantation de chacun a eu par la suite un effet fort structurant sur le développement de leur localités d'accueil et sur l'ensemble de la région, notamment avec l'implantation à leur voisinage de nombreux autres établissements hôteliers de divers types et catégories qui amplifiaient d'autant la force d'appel de ces lieux. L'hôtellerie n'a pas attendu l'apparition des centres commerciaux (ou centres d'achats, selon l'anglicisme courant) pour saisir les avantages de la concentration de plusieurs commerces de même nature dans un même lieu. Depuis des temps lointains, les hôtels vivent des bénéfices de la concurrence. D'instinct, à moins qu'il n'ait l'intention au préalable de loger dans un établissement en particulier, le voyageur se dirigera vers une destination qui lui présente choix et variété. Outre l'impression qu'il s'agit de toute évidence d'une destination intéressante, il en tire la conviction qu'il pourra ainsi comparer et trouver ce qu'il désire au prix qu'il veut payer. L'expérience démontre que tous en profitent: les hôteliers, la clientèle, tout comme le lieu d'accueil, car la diversité s'inscrit alors comme un atout; et comme un facteur d'attraction et de structuration supplémentaire.

Il faudra un jour évaluer à quel point cette hôtellerie et toutes les infrastructures qu'elle a drainées dans son sillage ont transformé le visage des Laurentides que le curé Labelle et consorts vouaient à la colonisation et au «salut de la race», donc à une autre structure de développement, à un autre aménagement de l'espace, à une autre économie. Chose sûre, elles ont favorisé la découverte, sous un angle différent et avec d'autres yeux, de la nature de cette portion des Laurentides et, au premier chef, de ses lacs, le tout presque à un jet de pierre d'une ville, Montréal, qui prenait une expansion accélérée, densifiait de plus en plus son tissu et alimentait chez nombre de ses habitants davantage favorisés des aspirations à s'évader hors du décor urbain. Autrement dit, l'hôtellerie et ses infrastructures ont eu une influence sensible sinon déterminante dans la prolifération d'une villégiature reposant sur une armature de chalets, d'abord d'été puis quatre-saisons, au fil des décennies.

L'autoroute 15, dite des Laurentides, est la manifestation la plus tangible de cette réalité. Celle-ci n'a pas été planifiée ni aménagée pour satisfaire les besoins des gens qui vivaient au quotidien dans les Laurentides de Montréal, mais plutôt pour répondre à l'afflux sans cesse croissant d'usagers temporaires et réguliers de ce territoire, à savoir les clients de l'hôtellerie qui avait essaimé et qui s'était fortifiée, à savoir également les propriétaires et les locataires de chalets. Cette infrastructure est née d'un besoin exogène qu'avait nourri depuis longtemps et de façon continue une population qui n' appartenait pas en propre à la région où elle s'est répandue.

\section{Le paradoxe touristique}

Nous touchons ici un élément-clé du paradoxe touristique : le tourisme tend en effet à banaliser les destinations. Attirés par l'ailleurs, les touristes font en sorte que cet ailleurs leur ressemble dans la mesure où leurs besoins et leurs attentes soient comblés : il leur faut des lieux où se restaurer, se divertir, se reposer, être hébergés. Des lieux qui, fondamentalement, ne les dépaysent pas trop. D'où l'édification un peu partout sur la planète, surtout en des destinations qui reposent sur l'exploitation de ressources naturelles telles que l'eau, le soleil ou la neige, d'un paysage «touristique » fortement façonné par la prépondérance, en hauteur comme en surface, d'établissements hôteliers beaucoup plus à l'image des régions émettrices que des sites récepteurs. À ce point de vue, la plupart des fronts de mer, qu'ils soient au Yucatan, sur la Riviera, en Floride ou sur la Costa Brava, sont la déclinaison d'un même substrat. Il en est de même pour les stations de montagne.

Un autre paradoxe en découle : la maîtrise du développement régional induit par le tourisme et plus spécifiquement par l'hôtellerie échappe souvent aux forces vives de la destination et, parfois même, à la pleine autorité des paliers gouvernementaux. La station touristique de Tremblant, qui de plus en plus s'impose comme un îlot distinct dans les Laurentides de Montréal, en est un exemple pertinent.

Terminons ce trop bref exposé en Méditerranée. Cette vaste mer intérieure, coincée entre le détroit de Gibraltar et les terres du Levant, qui baigne à la fois les côtes de l'Europe et de l'Afrique, est la première destination du globe. Selon les données officielles de l'Organisation mondiale du tourisme $(\mathrm{OMT})^{3}$, elle a reçu 219,6 millions de touristes internationaux en 1999, une augmentation de 4,7\% sur l'année précédente. Le mouvement ne semble pas vouloir ralentir: ils seront 350 millions en 2020.

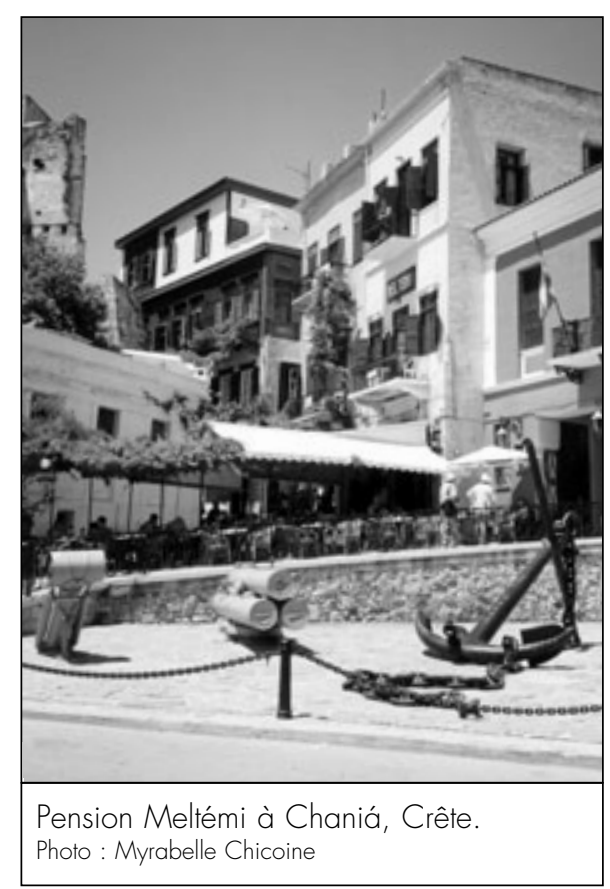


À l'heure actuelle, $84 \%$ d'entre eux proviennent du continent européen, surtout d'Allemagne, d'Espagne, de France, d'Italie et des pays scandinaves. Ce tourisme international sur le pourtour de la Méditerranée - qui ne comprend pas les déplacements des nationaux, tels les Parisiens qui vont sur la Côte d'Azur ou les Catalans qui se rendent sur la Costa del Sol - a engendré cette même année le tiers des 131,8 milliards de dollars américains que constitue l'ensemble des retombées économiques mondiales. Autre information de poids, notons que de 1997 à 1999 plus de $65 \%$ des revenus générés par le tourisme en Méditerranée sont allés dans les goussets d'une dizaine d'entreprises établies dans les principaux pays émetteurs, entreprises qui possèdent ou contrôlent transporteurs, voyagistes et structures hôtelières.

\section{Conclusion}

Un rapide survol de quelques exemples tirés du Maroc, des Antilles, du Club Med, de Charlevoix, des Laurentides de Montréal et du bassin méditerranéen nous a permis de constater que le développement touristique structurellement agencé autour de l'hôtellerie profite certes à l'économie des destinations et au développement régional, mais de manière bien imparfaite en raison de deux facteurs majeurs :

- sa dynamique fondamentale relève davantage des intérêts de touristes eux-mêmes et de ceux qui les font voyager que de ceux qui les accueillent ;

- le manque d'une intervention aménagiste intégrée nuit à une organisation équilibrée de l'espace et à une utilisation durable des ressources.

Sur ce dernier point, est révélatrice l'ignorance réciproque qu'ont entretenue au Québec - et qui perdure largement - les diverses générations de plans de développement touristique des associations touristiques régionales (ATR) et de schémas d'aménagement régional des municipalités régionales de comté (MRC). Chacune des parties y est allée de ses propres priorités et a fonctionné comme si elle existait en un univers distinct sans corrélations ni mutuelles influences avec l'autre.
C'est pourquoi l'expérience en cours aux îles de la Madeleine vaut d'être relevée. Dans cet archipel, l'expansion hôtelière a été freinée par la courte période d'affluence touristique : à l'exception du Madeli et du Château Madelinot qui offrent chacun une centaine de chambres à Cap-aux-Meules, le stock d'hébergement se distribue dans des auberges de faible capacité, dans des gîtes et surtout dans des chalets et des résidences principales loués aux visiteurs de juin à septembre.

N'empêche, la présence de 5000 à 6000 mille personnes de plus chaque jour dans les îles lors de la pointe estivale aux côtés des 13000 résidants permanents apporte son lot d'irritants, sinon de problèmes à plus ou moins long terme : pertes d'espaces agricoles et forestiers, pressions sur l'approvisionnement en eau potable et sur le traitement des déchets, hausse des propriétés et des valeurs foncières, prolifération d'équipements de «mauvais goût » et impacts sur le paysage, émergence d' «attitudes négatives » chez les Madelinots. Autant de problèmes liés au développement régional et à l'aménagement du territoire.

En mars 2004, quelque 225 personnes de tous horizons ont participé au Forum sur la politique cadre du développement touristique de l'archipel, tenu dans la foulée d'un colloque précédent en novembre 2002 organisé par l'Association touristique régionale. Les membres de l'ATR avaient alors affirmé « le caractère unique des îles et la nécessité d'en assurer un développement durable, sain et harmonieux ${ }^{4} \gg$ en privilégiant, sous l'égide de la MRC, l'adoption d'une telle politique cadre.

À cet égard, les Madelinots font un travail de pionniers. Les orientations et les décisions qu'ils prendront feront, en quelque sorte, œuvre de jurisprudence en matière de développement touristique. Leur vision aurait pu être sectorielle et ne se confiner qu'au tourisme. Au contraire, il leur est apparu dès le départ que les « balises » du développement touristique doivent relever d'une approche aménagiste, donc plus globale, et s'inscrire, en conséquence, au sein du schéma d'aménagement du territoire.
Normand Cazelais est géographe à Hydro-Québec et journaliste au Devoir.

\section{Notes}

1 Pour une analyse plus pointue du rôle des auberges dans l'évolution du tourisme et de l'hôtellerie au sein de la région, lire Cazelais, 1998 : 51-52).

2 Pour de plus amples renseignements sur les différents établissements, se référer aux deux tomes de Garceau (1990 et 1995).

3 OMT (2003), Tendances des marchés touristiques- Europe, 405 p.

4 Association touristique régionale des Îles de la Madeleine, Le tourisme aux Îles-de-laMadeleine: En planifier et en gérer la croissance en vue d'en assurer un développement harmonieux.

\section{Bibliographie}

Cazelais, Normand (1998), «Charlevoix et ses auberges », Téoros, vol. 17, nº 1, p. 51-52.

Dubé, Philippe (1986), Deux cents ans de villégiature dans Charlevoix, Les Presses de l'Université Laval, Québec.

Durrande Moreau, Agnès (2003), Services et développement régional, $\mathrm{XIII}{ }^{\circ}$ Conférence internationale du RESER (Réseau européen services et espace), Mons.

Garceau, Henri-Paul (1990). Chronique de l'hospitalité hôtelière du Québec, tome I, Les Publications du Québec / Le Méridien.

Garceau, Henri-Paul (1995), Chronique de l'hospitalité hôtelière du Québec, tome II, Éditions XYZ.

Monod, Jérôme et de Castelbajac, Philippe (1971), L'aménagement du territoire, Presses Universitaires de France, collection «Que Sais-Je ? », nº 987, Paris.

Paci, Enzo (1993), A Report on Global Tourism: Evaluating the Current Reality, Travel Review Conference, Saint John.

WTTO - World Travel and Tourism Organization / International Hotel and Restaurant Association (1999), Tourism and Sustainable Development - The Global Importance of Tourism, Background Paper \#1, Commission on Sustainable Development, Seventh Session, New York. 